\title{
Evaluation, Validation, and Recognition of the Point-of-Care Circulating Cathodic Antigen, Urine-Based Assay for Mapping Schistosoma mansoni Infections
}

\author{
Daniel G. Colley, ${ }^{1,2 \star}$ Charles H. King, ${ }^{3}$ Nupur Kittur, ${ }^{1}$ Reda M. R. Ramzy, ${ }^{4}$ William Evan Secor, ${ }^{5}$ Merlene Fredericks-James, ${ }^{6}$ \\ Giuseppina Ortu, ${ }^{7}$ Michelle N. Clements, ${ }^{8,9}$ Eugene Ruberanziza, ${ }^{10}$ Irenee Umulisa, ${ }^{10,11}$ Udo Wittmann, ${ }^{8,12}$ and
} Carl H. Campbell Jr.

${ }^{1}$ Schistosomiasis Consortium for Operational Research and Evaluation (SCORE), Center for Tropical and Emerging Global Diseases, University of Georgia, Athens, Georgia; ${ }^{2}$ Department of Microbiology, University of Georgia, Athens, Georgia; ${ }^{3}$ Center for Global Health and Diseases, Case Western Reserve University, Cleveland, Ohio; ${ }^{4}$ National Nutrition Institute, General Organization for Teaching Hospitals and Institutes, Cairo, Egypt; ${ }^{5}$ Division of Parasitic Diseases and Malaria, Centers for Disease Control and Prevention, Atlanta, Georgia; ${ }^{6}$ Ministry of Health and Wellness, Castries, St. Lucia; ${ }^{7}$ Santé Publique France, Paris, France; ${ }^{8}$ Schistosomiasis Control Initiative, London, United Kingdom; ${ }^{9}$ Medical Research Council, Clinical Research Trials Unit, University College London, London, United Kingdom; ${ }^{10}$ Malaria and Other Parasitic Diseases Division, Neglected Tropical Diseases and Other Parasitic Diseases Unit, Rwanda Biomedical Center, Ministry of Health, Kigali, Rwanda; ${ }^{11}$ African Leaders Malaria Alliance, Dar-es-Salam, Tanzania; ${ }^{12}$ Consult AG Statistical Services, Zurich, Switzerland

\begin{abstract}
Efforts to control Schistosoma mansoni infection depend on the ability of programs to effectively detect and quantify infection levels and adjust programmatic approaches based on these levels and program goals. One of the three major objectives of the Schistosomiasis Consortium for Operational Research and Evaluation (SCORE) has been to develop and/or evaluate tools that would assist Neglected Tropical Disease program managers in accomplishing this fundamental task. The advent of a widely available point-of-care (POC) assay to detect schistosome circulating cathodic antigen (CCA) in urine with a rapid diagnostic test (the POC-CCA) in 2008 led SCORE and others to conduct multiple evaluations of this assay, comparing it with the Kato-Katz (KK) stool microscopy assay-the standard used for more than 45 years. This article describes multiple SCORE-funded studies comparing the POC-CCA and KK assays, the pros and cons of these assays, the use of the POC-CCA assay for mapping of $S$. mansoni infections in areas across the spectrum of prevalence levels, and the validation and recognition that the POC-CCA, although not infallible, is a highly useful tool to detect low-intensity infections in low-to-moderate prevalence areas. Such an assay is critical, as control programs succeed in driving down prevalence and intensity and seek to either maintain control or move to elimination of transmission of $S$. mansoni.
\end{abstract}

\section{INTRODUCTION}

The microscopy-based Kato-Katz (KK) thick smear fecal assay is highly specific and has been used widely for more than 45 years $^{1}$ to determine the prevalence and gauge the intensity of Schistosoma mansoni infections. Where prevalence levels are high, it performs well, although it requires stool collections and qualified technical personnel to prepare and read slides. However, in low-prevalence areas or after successful control interventions, because of its lack of sensitivity, the KK assay underestimates true local prevalence of infection in such settings. ${ }^{2,3}$ As prevalence has decreased in many places because of preventive chemotherapy with praziquantel (PZQ), it has become increasingly apparent that Neglected Tropical Disease (NTD) program managers need a more sensitive and more field-applicable assay for mapping S. mansoni infection than stool microscopy.

In December 2008, the Bill \& Melinda Gates Foundation (BMGF) funded the Schistosomiasis Consortium for Operational Research and Evaluation (SCORE; https://score. uga.edu) to conduct operational research on the control and elimination of S. mansoni and Schistosoma haematobium. One objective of this program was to develop and evaluate mapping and diagnostic tools that would help NTD program managers in their efforts to control schistosomiasis. Even earlier in 2008, while the BMGF was still considering the SCORE proposal, Dan Colley, principal investigator of SCORE,

${ }^{*}$ Address correspondence to Daniel G. Colley, Schistosomiasis Consortium for Operational Research and Evaluation, University of Georgia, 145 Coverdell Center, 500 D.W. Brooks Dr., Athens, GA 30602. E-mail: dcolley@uga.edu and BMGF officers Julie Jacobson and Debbie Burgess met regarding the pending commercialization and availability of a point-of-care (POC) cassette assay that could detect circulating cathodic antigen (CCA) from $S$. mansoni in urine specimens. Circulating cathodic antigen is a genus-specific glycan antigen discovered in the mid-1970s that is vomited into the blood by adult schistosome worms living in blood vessels and is excreted in the urine of the mammalian host. ${ }^{4}$ An earlier rapid diagnostic test detecting CCA appeared to have great promise as a mapping tool for $S$. mansoni infection, ${ }^{5}$ but that assay was not widely commercialized for programmatic use and was later unavailable. Subsequently, a strong case was made for using rapid diagnostic tests for schistosomiasis mapping. ${ }^{6}$ In 2008/2009, the POC-CCA assay became widely available, but had not been widely used, especially in settings of different prevalence levels or in side-by-side comparisons with KK stool microscopy. Also, when such comparisons had been performed, the KK assay was almost always treated as a "gold standard," despite the logical inappropriateness of doing so, given its low sensitivity at lower levels of prevalence.

Propitiously, sales of the POC-CCA assay by Rapid Medical Diagnostics (RMD; Pretoria, South Africa) began in September 2008. Further evaluation of the POC-CCA test was included in the SCORE proposal to the BMGF because of the potential for this assay to be an important tool for mapping S. mansoni infections.

This article describes the various studies and evaluations conducted by SCORE to assess field performance of the POC-CCA assay. The evaluations included the SCORE fivecountry study, conducted in 2010; several focused studies conducted in Kenya; a systematic review using publications 
up to June 2015 of the KK-POC-CCA relationship; countrywide mapping studies in places with low and very low or no prevalence; and considerations regarding trace readings. It also describes remaining issues related to quantitation of infection intensity and POC-CCA batch variability, as well as SCORE's contributions to guidance and guidelines by the World Health Organization (WHO). Note that SCORE has had continued interest in the POC-CCA assay, but it has never had any financial link to the manufacturer or sellers of the commercial version of the POC-CCA test.

\section{FIRST EVALUATIONS OF THE POC-CCA ASSAY FOR MAPPING OF S. MANSONI: THE FIVE-COUNTRY STUDY}

To better understand POC-CCA performance, in September 2009, SCORE developed a request for applications (RFA) with input from its Advisory Committee and in consultation with WHO/NTD and WHO/AFRO. The RFA requested proposals to compare the POC-CCA assay with the KK assay performed on one, two, or three stools, with two slides per stool, for the detection of $S$. mansoni eggs from schoolchildren. These parallel evaluations were to be carried out in sites thought to have low (10-24\%) or moderate prevalence (25-50\%) for S. mansoni infections, or areas thought to have mixed S. mansoni and S. haematobium infections with the prevalence of each species estimated to be $\geq 25 \%$. The primary question was, "Is the POC-CCA assay just as good as the KK?" The primary outcome was to be the non-inferiority comparison of a single urine POC-CCA assay with the $\mathrm{KK}$ assay results from the two slides from the first of the three sequentially collected stools. This comparator was chosen to simulate the single stool sample KK testing used by most control programs, rather than the standard research use of three stool specimens. The results comparing a POC-CCA single urine assay with the KK assay data from all three collected stools were also analyzed, so as to evaluate the POC-CCA against KK assessments that would have increased sensitivity.

SCORE shared the RFA with 16 groups of investigators and received 13 proposals, of which five were funded. The five funded projects were based in Cameroon, Côte d'Ivoire, Ethiopia, Kenya, and Uganda. The sites in both Côte d'Ivoire and Cameroon had some level of mixed infections with S. haematobium, although the predominant species was S. mansoni.

Each of the five programs that were part of this project analyzed and subsequently published their own data. ${ }^{7-11}$ In some studies, both the prevalence and the intensity of $S$. mansoni infections were assessed. Eggs per gram (EPG) of feces reflected the intensity of infection for KK. Relative intensity of infection was scored with the POC-CCA by comparing the test band with the control band to give a score of trace (considered as a weak positive), $1+, 2+$, or $3+.{ }^{11}$ The SCORE secretariat undertook the analyses and publication of the overall combined data with the other Five-Country Study consortium members. ${ }^{12}$

Point-of-care-CCA diagnostic accuracy was further investigated by stool-based real-time polymerase chain reaction analyses on 905 stools selected from among the fivecountry study specimens, with oversampling of specimens from study participants who had discordant POC-CCA and $\mathrm{KK}$ results. This additional assay on some of the specimens provided another data set for the subsequent latent class analyses (LCAs) of POC-CCA diagnostic accuracy. ${ }^{12}$ In addition, the project in Ethiopia included data from an area of Ethiopia without schistosomiasis to determine whether the POC-CCA assay would yield false-positive results in a nonendemic area. POC-CCA tests of 100 children in this area were all negative, except for one trace result. KK assays on stools of these children were uniformly negative for S. mansoni, but $50 \%$ of them were positive for at least one soil-transmitted helminth. $^{12}$

Data from all 4,405 children with parallel KK and POC-CCA results demonstrated that the $\mathrm{POC}-\mathrm{CCA}$ urine assay was just as good as the KK assay for mapping $S$. mansoni infections and better in many circumstances. The urine specimens were easier to collect than stool, and the assay was much simpler to perform. Based on LCA, the POC-CCA was more sensitive (86\% versus $62 \%$ ) than $\mathrm{KK}$ but less specific $(72 \%$ versus $\sim 100 \%$ ) than duplicate KK smears from one stool. The sensitivity of the POC-CCA was also much better than the KK assay for infection intensities of $<100 \mathrm{epg}$. The relationship between POC-CCA and KK assays varied by prevalence: prevalence of $50 \%$ by KK corresponded with the prevalence by POC-CCA of $72 \%$, whereas a $10 \% \mathrm{KK}$ prevalence was roughly equivalent to a prevalence of $46 \%$ by POC-CCA. Subsequent studies by SCORE and analyses by others have further characterized this nonlinear relationship. ${ }^{13,14}$

One concern had been that the POC-CCA would be too expensive for program use because it was a commercial product. At that time, the price per cassette was higher than that of the materials used to perform the KK. However, in another SCORE study, once all of the expenditures were considered, including the additional personnel time and return field visits required by $\mathrm{KK}$, the two tests were found to be comparable in cost. ${ }^{15}$ By the time of this writing in 2019 , the cost of the POC-CCA assay has decreased even further because of its more widespread use and purchases in bulk.

An unfortunate aspect of this five-country study was that along with the standard commercial POC-CCA assay kits supplied by the manufacturer (RMD) for the studies, RMD included an "experimental/lower sensitivity" POC-CCA assay thought by RMD to be an improvement over the standard assay. In reality, this "experimental" POC-CCA assay was decidedly inferior to the standard version for detecting lowintensity infections. The results using this substandard assay were intended to be analyzed in house and were not expected to be published. However, in several cases, results from this inferior/noncommercial version of the assay were published in the site-specific publications, ${ }^{7,8,10,11}$ which resulted in continued confusion about the overall performance of the POC-CAA.

Kenyan studies on variability of POC-CCA results. Although the five-country study demonstrated the potential utility of the POC-CCA assay for mapping of S. mansoni infections, many people, including those at the WHO making recommendations and guidelines for schistosomiasis control, continued to raise questions about its performance. Therefore, SCORE undertook several additional studies in Kenya related to POC-CCA assay performance to expand the database and further establish the rationale for its use to map S. mansoni infections. These assessments found no significant variability among the batches of the assays that they studied at that time, and that intra- and inter-reader variability was insignificant. Although there was day-to-day variability in POC-CCA readings (18\% of urines from the same individuals 
tested on multiple days), it was much less than day-to-day variability of the stool KK assays (48\% of the individuals)..$^{16}$ In children who were POC-CCA positive but KK negative based on three stools/two slides each, $47 \%$ became POC-CCA negative after treatment with a single dose of praziquantel (PZQ); 34\% of those remaining POC-CCA positive after one treatment became negative after a second $P Z Q$ treatment. ${ }^{16}$

Systematic review of the relationship between $\mathrm{KK}$ and POC-CCA. SCORE developed additional insight into the nonlinear relationship between KK and POC-CCA prevalence through a systematic review of all 19 published articles as of June 2015 that directly compared these assays. ${ }^{13}$ At a prevalence greater than $50 \%$ by $\mathrm{KK}$, the two assays yielded approximately the same prevalence in terms of programmatic considerations, although the POC-CCA prevalence was often somewhat higher. By contrast, based on 21 data sets from 11 applicable studies, when KK prevalence was $<50 \%$, the prevalence by POC-CCA was between 1.5-fold higher (at a higher prevalence) and 6-fold higher (at the lower prevalence). This POC-CCA-to-KK-positive result ratio increased as KK prevalence decreased. ${ }^{13}$ Five of the publications compared the intensity of infection by KK epg with the visual band density using the POC-CCA assay. There was a clear trend, with those with darker POC-CCA band readings having higher median stool epg than those with lower density visual bands. ${ }^{13}$ In addition, a recent publication has provided a scoring system to aid in visual grading of the intensity of the reaction band observed. ${ }^{17}$

\section{SCORE-SUPPORTED MAPPING STUDIES COMPARING THE POC-CCA ASSAY WITH THE KK ASSAY FOR MAPPING IN LOW TO VERY LOW PREVALENCE AREAS}

In addition to the five-country study, SCORE has supported several other comparisons of the KK assay and the POC-CCA assay in multiple countries, especially those considered to have low or no prevalence of S. mansoni. These include studies in Ecuador (non-endemic), Burundi, and Rwanda (prevalence based on sentinel site surveillance before remapping $<2 \%$ by KK), St. Lucia (considered no longer endemic), Egypt (area-wide prevalence in the Nile delta $<2 \%$ by $\mathrm{KK})$, and the previously mentioned 100 children in an area of Ethiopia that was non-endemic for schistosomiasis.

Prevalence mapping studies in Burundi and Rwanda. The mapping studies in Burundi and Rwanda were conducted to inform studies being planned on the elimination of S. mansoni in these countries. Burundi and Rwanda had had consistent annual mass drug administration (MDA) programs for 6 or 7 years. $^{18-20}$ The MDA implementation differed somewhat between the two countries, but in both, annual MDA of $P Z Q$ was performed in those areas determined to need it, and based on KK sentinel site monitoring, both countries had achieved $<2 \%$ prevalence. The SCORE mapping studies, which followed these MDA programs, were conducted collaboratively with the respective Ministries of Health (MOHs), the Schistosomiasis Control Initiative (SCl), and the END Fund and were originally designed to estimate nationwide $S$. mansoni prevalence by POC-CCA testing of a single urine specimen from each of 50 13- to 14-year-old children per school in each of 400 schools. In approximately half of those schools, the POC-CCA assays were also compared with $\mathrm{KK}$ stool testing results (one stool/two slides) from the same children who provided urines. In Burundi, prevalence by POC-CCA was $42.8 \%$ in the 17,331 children tested. ${ }^{19}$ Of these, 8,482 children were also tested using $\mathrm{KK}$, yielding a prevalence of $1.5 \%$ by the KK assay and $41.3 \%$ by the POCCCA assay. In Rwanda, testing similar numbers of children in the same ways yielded remarkably similar KK and POC-CCA data and analyses, with POC-CCA results indicating S. mansoni infections, albeit most of low intensity, in all 31 mapping units. ${ }^{20}$

A subset of urine specimens (398) from Burundi was selected from eight sentinel site schools to include a spectrum of prevalence levels, spanning $0-20 \%$ by the KK assay and $12-90 \%$ by the POC-CCA assay. These urine samples were sent to Leiden University Medical Center (LUMC) to be further tested by the very sensitive and schistosome-specific upconverting phosphor lateral flow circulating anodic antigen (UCP-LF CAA) assay. ${ }^{21}$ In this selected subset, the average prevalence levels by KK, POC-CCA, and UCP-LF CAA were $6.8 \%, 53.5 \%$, and $46.5 \%$, respectively. Sixty-one percent of the positive POC-CCA readings were traces. Further analysis by LCA indicated that the POC-CCA assay outperformed the KK assay at the low infection intensities in Burundi. Latent class analysis estimated that approximately $50 \%$ of trace readings were true positives. ${ }^{22}$ Furthermore, it was estimated that the KK assay missed $\sim 85 \%$ of infections, albeit most of those were likely of light intensities or egg negative. Again, the Rwanda data and LCA analyses provide conclusions very similar to those from the Burundi mapping. ${ }^{20}$

It is clear based on the mapping studies in Burundi and Rwanda that even when prevalence is very low by $\mathrm{KK}$, it is much higher by POC-CCA. It is also clear that most of those who are KK negative and POC-CCA positive have trace readings. SCORE has referred to the many individuals in lowto-moderate prevalence areas with positive POC-CCA results and no eggs found in stools (at least by the KK assay on two slides from one stool) as "egg-negative/worm-positive" (see in the following text).

Mapping study in St. Lucia. At one time, the Caribbean island nation of St. Lucia was highly endemic for S. mansoni. However, following the Research and Control Project on schistosomiasis (1967-1981) ${ }^{23}$ and during considerable economic development in the intervening 40 years to the present, schistosomiasis seems to have disappeared. In collaboration with the Ministry of Health and Wellness of the Government of St. Lucia, the Pan American Health Organization, and the Centers for Disease Control and Prevention (CDC), SCORE assisted in mapping study of all primary schools $(n=63)$ on St. Lucia. ${ }^{24}$ This mapping included $16 \%$ of the 8,985 children aged between 8 and 11 years on the island and included collection of urine $(n=1487)$ and finger-stick blood ( $n=1455)$ samples. Fourteen percent $(n=209)$ of the children providing a urine sample had a trace $(n=150)$ or $1+$ $(n=59)$ POC-CCA result in the field. Some of the samples were also reassessed as trace or $1+$ readings when retested by POC-CCA at the University of Georgia. However, on subsequent testing of suspected positive urines by the UCP-LF CCA assay at LUMC, although there were a few, very low, inconsistently positive results on multiple UCP-LF CAA tests, they were not from the same urines that had low positive POCCCA values. Similarly, although there were some children $(n=$ 8; $0.6 \%$ ) with initial anti-schistosome-soluble egg antigen ELISA antibody results slightly higher than the cutoff, none tested positive for schistosome infection by confirmatory 
western blot using the S. mansoni adult microsomal antigen. ${ }^{25}$ Furthermore, there was no correlation among the children who tested positive by POC-CCA, those who tested positive by the UCP-LF CAA, or those who were initially positive by anti-SEA ELISA. The UCP-LF CAA assay and the western blot assay are considered by many as confirmatory assays. Therefore, we concluded that negative and inconclusive results using those two assays meant that none of the children in the study was confirmed to have S. mansoni infection. ${ }^{24}$

\section{INTERPRETING TRACE-POSITIVE READINGS OF THE POC-CCA ASSAY}

It is clear based on the data from very low-prevalence settings that an important challenge is how to read and interpret visually faint bands, called "trace." The manufacturer's instructions stated that trace should be considered as positive, and, eventually, most of the evidence generated by LCAs and other studies led the $\mathrm{WHO}$ to also state that trace results should be considered positive. This appears to be appropriate, except in areas with extremely low prevalence-around $1-2 \%$ by KK. Although not every trace reading is a true positive, when the POC-CCA is used for mapping purposes to determine MDA interventions, somewhat overestimating prevalence by scoring trace results as positive can ensure that areas with infected individuals are not left untreated.

SCORE made attempts to use smartphone and tablet apps as quantitative readers to overcome the challenge of both the trace readings and the subjective interpretation of the intensity of the "test line" compared with the intensity of the "control" band. Although the applications achieved the goal of providing quantitative readings of POC-CCA band intensity, the goal of distinguishing between trace results that are false positive versus true positive has thus far not been achieved.

How can someone be KK egg-negative and POCCCA-positive? There are multiple possible explanations for the discrepancy sometimes observed between a negative KK and a positive POC-CCA result, especially when the POCCCA readings are trace or $1+$. One is that the two assays measure different schistosome life stages. The KK measures only eggs that are excreted in the feces, whereas the POCCCA detects a product from living adult worms excreted in the urine. Furthermore, the relationship between the number of worms and the number of eggs excreted at any given time point during this yearslong infection is not known. In fact, it is likely that the relationship between eggs and adult worms (and thus CCA production) changes over time during this chronic infection. ${ }^{26}$ When both assays are used appropriately by trained users, some of the possible reasons for egg-negative CCA-positive schistosomiasis are as follows:

1. The KK assay is insensitive and missed an egg.

a) The egg was in another part of the stool.

b) The egg was excreted on a different day.

2. The POC-CCA result was a false positive.

3. The POC-CCA readers/technicians were insufficiently trained or trained differently in different programs/teams and read a negative result as a positive.

4. The person harbors a bisexual infection, but the female worms became infertile.

5. The person harbors a bisexual infection, but anti-fecundity immunity reduced or stopped egg production.
6. The person harbors a single sex infection.

What should an NTD program manager do when confronted with a person who is egg negative/POC-CCA positive, particularly when there are few individuals with POC-CCA readings greater than $1+$ and an overall prevalence by the KK assay that is very low? This issue arose in the Burundi ${ }^{19}$ and Rwanda studies, ${ }^{20}$ where prevalence in some villages was very low or zero by $\mathrm{KK}$, but many children were POC-CCA positive, albeit with a preponderance of trace readings (see in the following text). If these children had worms that were excreting eggs, albeit at low levels, they still could be at risk for morbidity or pose a risk for transmission.

Regardless of the reason for someone being egg negative/ POC-CCA positive, understanding the answer to the question "If the worms are not making eggs, are they causing morbidity?" has important implications for control of morbidity and elimination activities.

Studies of POC-CCA positives in areas of very low prevalence in Egypt. SCORE collaborated with the Ministry of Health and Population of the government of Egypt to conduct an intensive evaluation of egg excretion from children in an area of very low schistosomiasis prevalence by $\mathrm{KK}(<2 \%)$. These children lived in three districts that had been under schistosomiasis control for many decades. ${ }^{27,28}$ The three districts chosen for the SCORE studies of egg excretion had prevalence levels of $1.2 \%, 0.0 \%$, and $0.9 \%$ by the KK assay based on more than 2,000 children tested in a mapping study in $2016 .^{27}$

In late 2017 , the study enrolled 45 children who had POCCCA results of trace or $1+$ but who were KK negative on initial screening. The primary study question was whether such eggnegative/trace or 1+ POC-CCA-positive children in this area of very low prevalence excrete detectable S. mansoni eggs over a 30-day period. Stool and urine samples were collected every day from each child for 30 days. Stool samples were examined by the KK assay (one stool/four slides), and all S. mansoni egg-negative stools were further tested by the miracidia hatching test (MHT). Daily urine specimens were examined by one POC-CCA assay. The data clearly indicated those KK egg-negative children with trace or 1+ POC-CCA readings very infrequently (one of 1,388 stools; $0.1 \%$ ) pass S. mansoni eggs. ${ }^{29}$ Thus, such children are unlikely to have ongoing egg-focused morbidity or contribute to the transmission of schistosomiasis.

To evaluate whether these children harbored low, undetectable numbers of adult worms or the POC-CCA results in this setting were false positives, SCORE's Egyptian collaborators investigated whether the trace or 1+ POC-CCA readings of KK egg-negative children would change to negative POC-CCA following one, two, or three treatments with PZQ. Of the 45 children in the 30-day study of stools and urine, 44 participated in this follow-up treatment study. ${ }^{30}$ The first and second PZQ treatments were conducted 3 months apart, and 5 weeks separated the second and third PZQ treatments. Stool and urine specimens were collected 3 months following the initial PZQ treatment, 3 weeks following the second PZQ treatment, and then 3 weeks after the third PZQ treatment. For each evaluation, stool and urine specimens were collected on three successive days. Stool specimens were examined by the KK assay (one stool/four slides), and all S. mansoni eggnegative stools were further tested by the MHT. Each urine 
sample was examined by one POC-CCA. Over the study period, all stool samples from study subjects remained S. mansoni egg-negative by $\mathrm{KK}$ and $\mathrm{MHT}$. Of the POC-CCA test results on the first 3 days of urine collections 3 months following the initial treatment, $29.6 \%$ were negative, $61.4 \%$ had trace-positive POC-CCA results, and 9.1\% had POCCCA 1+ results. Following the two additional PZQ treatments, the POC-CCA test results fluctuated between negative, trace, and $1+$, but did not consistently become negative. Furthermore, there were no differences between the proportions of POC-CCA trace and 1+ results obtained in the first day $(70.5 \%)$ and on the last day of the study $(72.7 \%)$. The lack of consistent change in test results to negative after multiple treatments makes it likely that the trace and 1+ POC-CCA readings in this very low prevalence area that had received control interventions for decades were false positives. ${ }^{30}$ We conclude that these children are neither at risk for schistosomiasis-related morbidity nor do they represent a public health problem in terms of contributing to the transmission of schistosomiasis.

The challenges of interpreting trace POC-CCA readings in different places. Based on the LCA studies of the data from the SCORE mapping in Burundi and Rwanda, approximately $50 \%{ }^{20,22}$ or at least $50 \%$ of the trace POC-CCA readings were estimated to be true positives. ${ }^{20-22}$ In mapping settings like these, where MDA had been going on for only 6 or 7 years and prevalence is low by the KK assay but high by POC-CCA, we propose categorizing trace results as positive. This would ensure treatment is provided to people in areas that would benefit by treatment but that would be excluded based on KK mapping. However, in areas that are nearing or perhaps have achieved elimination, for example, in St. Lucia and the areas in Egypt where the aforementioned studies were conducted and control interventions have been going on for many decades, it appears that trace POC-CCA readings are very likely to all be false positives. ${ }^{24,29,30}$

In deciding whether POC-CCA trace readings should be interpreted as false or true positives, we propose that both the control history and current prevalence of the location should be considered. As indicated earlier, the three villages in the Egyptian studies had prevalence levels of $1.2 \%, 0.0 \%$, and $0.9 \%$ by the KK assay based on more than 2,000 children tested. In the same survey, the POC-CCA prevalence in these villages, testing the same children, was $9.8 \%, 10.8 \%$, and $7.6 \%$, respectively, ${ }^{27}$ and almost $90 \%$ of those read as positive by POC-CCA were trace or 1+ readings. Furthermore, the mapping in St. Lucia ${ }^{24}$ was performed more than three decades after extensive interventions were applied across the country, and St. Lucia had undergone widespread development with much of the country moving from an agricultural-based to a tourism-based economy. ${ }^{31,32}$ It seems likely that any schistosomiasis transmission dynamics encountered in St. Lucia and the three study villages in Egypt would differ considerably from those taking place in Burundi and Rwanda, each of which had undergone selective area MDA with PZQ for only 6 years at the time of the mapping surveys. ${ }^{18,33,34}$ Another difference in the two different types of settings (Table 1) is the proportion of children with trace POC-CCA readings (Egypt $=8.4 \%$; St. Lucia $=10 \%$ compared with Burundi $=27 \%$; Rwanda $=30 \%$ ).

We hypothesize that in areas where transmission is clearly continuing, albeit perhaps at relatively low levels in most areas, such as in Burundi and Rwanda, there are many more low-level infections that are not detected by the KK assay but are detected by trace and $1+$ POC-CCA readings, and at least $50 \%$ of these are true positives. This hypothesis could be tested in Burundi, Rwanda, or other similar locations by evaluating the clearance (or lack thereof) of CCA following PZQ treatments. ${ }^{16,30}$ By contrast, in locations where multiple control interventions and/or development have been going on for decades, a child who is KK negative and POC-CCA trace or $1+$ reading, in the face of either extremely low or no transmission, is almost certainly false positive, as described earlier with the studies in Egypt and St. Lucia. Any test that has less than $100 \%$ specificity will begin to have larger percentages of false-positives as the true infection level goes down. Therefore, we propose that if the prevalence by POC-CCA in a given area goes below a given threshold, perhaps $8 \%$ or $10 \%$, and all readings are trace or $1+$, and there is no other evidence of schistosome transmission (either by $\mathrm{KK}, \mathrm{MHT}$, or xenomonitoring of snails), the $\mathrm{POC}-\mathrm{CCA}$ readings are most likely false positives. At that point, confirmatory assays and surveillance schemes will be needed to monitor for sporadic, resurgent transmission.

TABLE 1

Comparison of proportion of children with trace POC-CCA readings in four different settings

\begin{tabular}{|c|c|c|c|c|c|}
\hline & & Egypt & St. Lucia & Rwanda & Burundi \\
\hline $\begin{array}{l}\text { Current data } \\
\text { Sampling }\end{array}$ & & $\begin{array}{l}465 \text { schoolchildren in three } \\
\text { districts surveyed with both } \\
\mathrm{KK} \text { and POC-CCA }\end{array}$ & $\begin{array}{l}1,487 \text { children from island- } \\
\text { wide mapping with POC- } \\
\text { CCA }\end{array}$ & $\begin{array}{l}\text { 8,697 children from country- } \\
\text { wide mapping who have } \\
\text { data on both KK and POC- } \\
\text { CCA }\end{array}$ & $\begin{array}{l}\text { 9,371 children from country- } \\
\text { wide mapping who have } \\
\text { data on both KK and POC- } \\
\text { CCA }\end{array}$ \\
\hline Source of $d$ & ata & Haggag et al. ${ }^{28}$ & $\begin{array}{l}\text { Mapping dataset from St. } \\
\text { Lucia }^{24}\end{array}$ & $\begin{array}{l}\text { Mapping dataset from } \\
\text { Rwanda }^{20}\end{array}$ & Ortu et al. ${ }^{19}$ \\
\hline KK positive & & $3(0.6 \%)$ & Not tested & $172(2.0 \%)$ & $157(1.7 \%)$ \\
\hline POC-CCA & Negative & $364(78 \%)$ & $1,278(86 \%)$ & $5,438(62.5 \%)$ & $5,508(58.8 \%)$ \\
\hline result & Trace & $39(8.4 \%)$ & $150(10 \%)$ & $2,513(28.9 \%)$ & $2,827(30.2 \%)$ \\
\hline & $1+$ & $48(10.3 \%)$ & $59(4 \%)^{\star}$ & $479(5.5 \%)$ & $648(6.9 \%)$ \\
\hline & $2+$ & $11(2.4 \%)$ & & $147(1.7 \%)$ & $204(2.2 \%)$ \\
\hline & $3+$ & $3(0.6 \%)$ & & $120(1.4 \%)$ & $184(2.0 \%)$ \\
\hline \multicolumn{6}{|c|}{ Treatment history in the area } \\
\hline & & $\begin{array}{l}\text { Schistosomiasis control for } \\
\text { many decades }\end{array}$ & $\begin{array}{l}\text { Three decades of extensive } \\
\text { interventions, and } \\
\text { development of a tourism- } \\
\text { based economy }\end{array}$ & $\begin{array}{l}\text { Selective praziquantel mass } \\
\text { drug administration for } 6 \\
\text { years before mapping } \\
\text { survey }\end{array}$ & $\begin{array}{l}\text { Selective praziquantel mass } \\
\text { drug administration for } 6 \\
\text { years before mapping } \\
\text { survey }\end{array}$ \\
\hline
\end{tabular}




\section{ADDITIONAL CONSIDERATIONS IN USING POC-CCA FOR MAPPING}

The lack of quantification provided by the POC-CCA assay. The visually observed band density of the POC-CCA only provides a semi-quantitative correlate with the egg count-based intensity reading that can be provided by KK. Nevertheless, there is a reasonable relationship between the KK EPG estimate and the intensity of the POC-CCA band, ${ }^{13}$ which can allow for a general estimate of the intensity of infection. Furthermore, given the variability of the quantitative egg count obtained by the KK assay at low-to-moderate levels of intensity, ${ }^{35}$ this may not be as critical a difference as perceived by some.

Variability in POC-CCA batches. As noted earlier, there were no significant differences among the batches of POCCCA assays evaluated in the studies by Mwinzi et al. ${ }^{16} \mathrm{How}-$ ever, this has not held true for some batches produced later by RMD and ICT Diagnostics (ICT; Noordhoek, South Africa) and sold to programs and researchers, for example, the "experimental/lower sensitivity" version of the test sent to investigators in the five-country study. In 2016, the company undertook "optimization" efforts to try to reduce the percentages of trace results. SCORE was involved in evaluating two optimization batches, referred to as Version 1 and Version 2. These evaluations were conducted on stored urine from three countries and on fresh urine specimens collected in Mwanza Region, Tanzania, in August and December 2016. The overall results from both optimized versions showed that, although specificity was higher, sensitivity was much lower than that of previous standard batches, and SCORE strongly recommended that these so-called by RMD optimized versions 1 and 2 not be distributed for mapping or study purposes. Nevertheless, RMD moved ahead and produced batches with less sensitivity, which they sold from December 2016 through March 2017. RMD and ICT failed to communicate this change in sensitivity to those who purchased their products during that period, compounding the confusion by those who used them. Thus, there are data from some studies that may not be useful nor comparable to most of the other studies conducted with the standard batches both in the same countries over time as well as between countries.

In October 2017, RMD made another change in the assay from requiring one drop of urine and one drop of buffer to requiring two drops of urine and no buffer. This change was announced to customers, and this is the version being sold to this day (spring 2019). This change was noted on the company's website and did not alter the specificity or sensitivity of the standard POC-CCA cassette assay. In parallel urine testing, the sensitivity of this two drops of urine/no buffer version of the POC-CCA assay is similar to that of the earlier (preDecember 2016) standard version. As a continued precaution, SCORE recommends that the batch numbers and expiration dates be recorded for all mapping and research purposes and reported with any results.

Aberrant results have also been reported related to the use of the POC-CCA assay in Brazil. ${ }^{36,37}$ Some of these results may be attributable to the fact that Brazil requires the assay to be assembled in Brazil using components from the South African supplier, which could result in different levels of quality control of the final product, ${ }^{37}$ or this could be related to the issue of different batches of components mentioned earlier.
Schistosoma haematobium and the POC-CCA. Unfortunately, the POC-CCA assay is inconsistent for the detection of infection with S. haematobium. ${ }^{38-41}$ Thus, because the standard urine filtration assay for $S$. haematobium eggs is, like the KK, insensitive at low-to-moderate levels of infection intensity, ${ }^{42,43}$ a tool is still needed for mapping S. haematobium in low prevalence areas.

\section{SCORE'S CONTRIBUTIONS TO WHO GUIDANCE AND RECOMMENDATIONS RELATED TO POC-CCA USE FOR MAPPING OF S. MANSONI INFECTIONS}

By the time the five-country study was published in early $2013,^{12}$ the individual country data and the combined data had been presented many times in many international venues, and findings that the POC-CCA was more sensitive and easier to do than the KK assay were widely known. At the SCORE Annual Meeting in May 2012, which included the SCORE Advisory Committee and two WHO/NTD representatives, SCORE was encouraged to draft a recommendation to the WHO/NTD office regarding the potential use of the POC-CCA. Subsequently, a draft statement was prepared and shared with all participants at the 2012 SCORE Annual Meeting for input. After multiple revisions, the final statement was sent to $\mathrm{WHO} /$ NTD on May 25, 2012. Providing data from the five-country study and noting that others had generated similar data, the final cover email and statement asked that the WHO take the following under advisement: "SCORE recommends that a single urine examination by the commercially available $\mathrm{POC} /$ CCA cassette-based test can be used-and in our estimation should be used-instead of a single stool examination by the $\mathrm{KK}$ method to assess the prevalence of $S$. mansoni infections in children of school age for the purposes of mapping S. mansoni for decision-making in regard to preventive chemotherapy." They never received a response from the WHO. An additional 3 years passed before this issue was addressed by the WHO and the NTD-STAG Global Working Group on Monitoring and Evaluation of Preventive Chemotherapy approved the POC-CCA "for use in monitoring and evaluation of $S$. mansoni infection control and elimination programs."

Recently, there has been a major modeling effort published regarding the relationship of the KK assay to the POC-CCA across the spectrum of prevalence levels. ${ }^{14} \mathrm{SCORE}, \mathrm{SCl}$, and the WHO assisted the investigators in compiling all the existing comparative data on these two assays. The resulting article describes the relationship between KK prevalence and different levels of POC-CCA prevalence and states the implications of this relationship for applying current WHO guidelines, which are based on KK prevalence levels, to results using POC-CCA. Based on this comprehensive analysis, the WHO is currently considering a recommendation relating these two assays, leading to a recommendation that it is acceptable to use the POC-CCA to evaluate the prevalence of $S$. mansoni. Paired recommendations for cutoffs based on CCA or KK prevalence levels will allow NTD program managers to use either assay to estimate the prevalence that would be expected using the other assay. It is anticipated that some programs in areas that still have high prevalence levels may continue to use the $\mathrm{KK}$, with which they are familiar, and which also provides an opportunity to assess soil-transmitted helminths. However, in low-to-moderate prevalence areas, the POC-CCA will clearly provide a better estimate of the 
actual prevalence of S. mansoni. Regardless of expected prevalence, the POC-CCA is an easier test to use because of the relative ease of collecting urine samples compared with stools and the faster time to obtaining results.

\section{OTHER SCORE EFFORTS RELATED TO DIAGNOSTIC TOOLS}

SCORE has also supported research to help develop an assay for both for S. mansoni and S. haematobium that would be both highly specific and highly sensitive. SCORE-supported work focused on the UCP-LF CAA assay and its use as a confirmatory assay. These efforts are described elsewhere in this supplement. ${ }^{44}$ In addition, because of the extreme need, SCORE also supported initial efforts to use CAA in the development of a rapid diagnostic ${ }^{44}$ that would be suitable for mapping both S. mansoni and S. haematobium.

\section{FINAL THOUGHTS ON SCORE'S EFFORTS TO EVALUATE THE USEFULNESS OF THE POC-CCA ASSAY FOR MAPPING S. MANSONI INFECTIONS AND DEVELOPMENT OF FUTURE DIAGNOSTIC TOOLS}

The widespread commercial availability of the POC-CCA assay for mapping of active $S$. mansoni infections coincided with the start of SCORE. This provided an excellent opportunity to evaluate this field-friendly assay and relate it to the standard diagnostic assay at the time, the KK. It should be reiterated that SCORE has never had any financial link to the manufacturers or distributors of the POC-CCA (RMD and ICT) and that all of the multiple SCORE-funded evaluations in all 12 countries involved in one study or another were performed solely to determine how it would perform as needed for mapping infection with S. mansoni.

SCOREs presentations and publications have consistently stated that considering the $\mathrm{KK}$ assay the gold standard in evaluations of specificity and sensitivity of the POC-CCA is inappropriate because of the known insensitivity of the $\mathrm{KK}$ assay, especially in areas with low-to-moderate prevalence. Nevertheless, some groups, including the Cochrane systematic reviews, continue to make such mistaken comparisons. ${ }^{45}$

Many groups have pursued multiple other diagnostic assays for detection of schistosome infections including using antibody assays that detect either current or past infections, nucleic acid amplification tests, as well as assays to detect other carbohydrates and proteins of schistosomes. Furthermore, SCORE has participated in at least five meetings and conference calls to develop suitable target product profiles (TPPs) for schistosomiasis diagnostics and has strongly advocated for the development needed to make these TPPs a reality for programs seeking to control and/or eliminate schistosomiasis. It is hoped that efforts will continue to develop and rigorously evaluate all such assays, and these efforts will lead to standardized, commercially available, fieldapplicable tools for mapping, elimination verification, surveillance, and individual diagnoses of schistosomiasis.

Received October 23, 2019. Accepted for publication February 1, 2020.

Published online May 12, 2020.

Acknowledgments: We are pleased to acknowledge the contributions made by the many communities, children, and their families and field and laboratory staff who participated in the studies summarized in this article.

Financial support: The work summarized herein received financial support from University of Georgia Research Foundation, Inc., which was funded by the Bill \& Melinda Gates Foundation for the SCORE project. In addition, a significant amount of praziquantel provided as treatment during many of the studies reported on was donated through the WHO, USAID, RTI, World Vision, and SCI.

Authors' addresses: Daniel G. Colley, Nupur Kittur, and Carl H. Campbell Jr., Schistosomiasis Consortium for Operational Research and Evaluation (SCORE), Center for Tropical and Emerging Global Diseases, University of Georgia, Athens, GA, E-mails: dcolley@ uga.edu, nkittur@uga.edu, and ccamp@uga.edu. Charles H. King, Center for Global Health and Diseases, Case Western Reserve University, Cleveland, OH, E-mail: chk@cwru.edu. Reda M. R. Ramzy, National Nutrition Institute, General Organization for Teaching Hospitals and Institutes, Cairo, Egypt, E-mail: reda.mr.ramzy@gmail.com. William Evan Secor, Division of Parasitic Diseases and Malaria, Centers for Disease Control and Prevention, Atlanta, GA, E-mail: was4@ cdc.gov. Merlene Fredericks-James, Ministry of Health and Wellness, Castries, St. Lucia, E-mail: mfredericks2020@yahoo.com. Giuseppina Ortu, Santé publique France, Paris, France, E-mail: giuseppina_ortu@ outlook.com. Michelle N. Clements, Medical Research Council, Clinical Research Trials Unit, University College London, London, United Kingdom, E-mail: michelle.clements@ucl.ac.uk. Eugene Ruberanziza, Malaria and Other Parasitic Diseases Division, Neglected Tropical Diseases and Other Parasitic Diseases Unit, Rwanda Biomedical Center, Ministry of Health, Kigali, Rwanda, E-mail: ruberanzizaeugene@ gmail.com. Irenee Umulisa, African Leaders Malaria Alliance, National Institute of Medical Research Complex (ALMA), Dar-es-Salam, Tanzania, E-mail: umulisa5@gmail.com. Udo Wittmann, Consult AG Statistical Services, Zurich, Switzerland, E-mail: udo.wittmann@gmx.ch.

This is an open-access article distributed under the terms of the Creative Commons Attribution (CC-BY) License, which permits unrestricted use, distribution, and reproduction in any medium, provided the original author and source are credited.

\section{REFERENCES}

1. Katz N, Chaves A, Pellegrino J, 1972. A simple device for quantitative stool thick-smear technique in schistosomiasis mansoni. Rev Inst Med Trop Sao Paulo 14: 397-400.

2. de Vlas SJ, Gryseels B, 1992. Underestimation of Schistosoma mansoni prevalences. Parasitol Today 8: 274-277.

3. de Vlas SJ, Gryseels B, van Oortmarssen GJ, Polderman AM, Habbema JD, 1993. A pocket chart to estimate true Schistosoma mansoni prevalences. Parasitol Today 9: 305-307.

4. Deelder AM et al., 1994. Quantitative diagnosis of Schistosoma infections by measurement of circulating antigens in serum and urine. Trop Geogr Med 46: 233-238.

5. Stothard JR, Kabatereine NB, Tukahebwa EM, Kazibwe F, Rollinson D, Mathieson W, Webster JP, FenwickA, 2006. Use of circulating cathodic antigen (CCA) dipsticks for detection of intestinal and urinary schistosomiasis. Acta Trop 97: 219-228.

6. Stothard JR, 2009. Improving control of African schistosomiasis: towards effective use of rapid diagnostic tests within an appropriate disease surveillance model. Trans $R$ Soc Trop Med Hyg 103: 325-332.

7. Adriko M, Standley CJ, Tinkitina B, Tukahebwa EM, Fenwick A, Fleming FM, Sousa-Figueiredo JC, Stothard JR, Kabatereine NB, 2014. Evaluation of circulating cathodic antigen (CCA) urine-cassette assay as a survey tool for Schistosoma mansoni in different transmission settings within Bugiri district, Uganda. Acta Trop 136: 50-57.

8. Coulibaly JT et al., 2011. Accuracy of urine circulating cathodic antigen (CCA) test for Schistosoma mansoni diagnosis in different settings of Cote d'Ivoire. PLoS Negl Trop Dis 5: e1384.

9. Erko B, Medhin G, Teklehaymanot T, Degarege A, Legesse M, 2013. Evaluation of urine-circulating cathodic antigen (urine-CCA) cassette test for the detection of Schistosoma mansoni infection in areas of moderate prevalence in Ethiopia. Trop Med Int Health 18: 1029-1035. 
10. Tchuem Tchuente LA, Kuete Fouodo CJ, Kamwa Ngassam RI, Sumo L, Dongmo Noumedem C, Kenfack CM, Gipwe NF, Nana ED, Stothard JR, Rollinson D, 2012. Evaluation of circulating cathodic antigen (CCA) urine-tests for diagnosis of Schistosoma mansoni infection in Cameroon. PLoS Negl Trop Dis 6: e1758.

11. Foo KT, Blackstock AJ, Ochola EA, Matete DO, Mwinzi PN, Montgomery SP, Karanja DM, Secor WE, 2015. Evaluation of point-of-contact circulating cathodic antigen assays for the detection of Schistosoma mansoni infection in low-, moderate-, and high-prevalence schools in western Kenya. Am J Trop Med Hyg 92: 1227-1232.

12. Colley DG et al., 2013. A five-country evaluation of a point-of-care circulating cathodic antigen urine assay for the prevalence of Schistosoma mansoni. Am J Trop Med Hyg 88: 426-432.

13. Kittur N, Castleman JD, Campbell CH Jr., King CH, Colley DG, 2016. Comparison of Schistosoma mansoni prevalence and intensity of infection, as determined by the circulating cathodic antigen urine assay or by the Kato-Katz fecal assay: a systematic review. Am J Trop Med Hyg 94: 605-610.

14. Barenbold $O$ et al., 2018. Translating preventive chemotherapy prevalence thresholds for Schistosoma mansoni from the KatoKatz technique into the point-of-care circulating cathodic antigen diagnostic test. PLoS Negl Trop Dis 12: e0006941.

15. Worrell CM, Bartoces M, Karanja DM, Ochola EA, Matete DO, Mwinzi PN, Montgomery SP, Secor WE, 2015. Cost analysis of tests for the detection of Schistosoma mansoni infection in children in western Kenya. Am J Trop Med Hyg 92: 1233-1239.

16. Mwinzi PN, Kittur N, Ochola E, Cooper PJ, Campbell CH Jr., King $\mathrm{CH}$, Colley DG, 2015. Additional evaluation of the point-ofcontact circulating cathodic antigen assay for Schistosoma mansoni infection. Front Public Health 3: 48.

17. Casacuberta-Partal M, Hoekstra PT, Kornelis D, van Lieshout L, van Dam GJ, 2019. An innovative and user-friendly scoring system for standardised quantitative interpretation of the urinebased point-of-care strip test (POC-CCA) for the diagnosis of intestinal schistosomiasis: a proof-of-concept study. Acta Trop 199: 105150.

18. Ortu G et al., 2016. The impact of an 8-year mass drug administration programme on prevalence, intensity and co-infections of soil-transmitted helminthiases in Burundi. Parasit Vectors 9: 513.

19. Ortu G et al., 2017. Countrywide reassessment of Schistosoma mansoni infection in Burundi using a urine-circulating cathodic antigen rapid test: informing the National Control Program. $A m$ J Trop Med Hyg 96: 664-673.

20. Ruberanziza E et al., 2020. Nationwide remapping of Schistosoma mansoni infection in Rwanda using Circulating Cathodic Antigen Rapid Test: taking steps toward elimination. Am J Trop Med Hyg. 103: 315-324.

21. Sousa MS, van Dam GJ, Pinheiro MCC, de Dood CJ, Peralta JM, Peralta RHS, Daher EF, Corstjens P, Bezerra FSM, 2019. Performance of an ultra-sensitive assay targeting the circulating anodic antigen (CAA) for detection of Schistosoma mansoni infection in a low endemic area in Brazil. Front Immunol 10: 682.

22. Clements $M N$ et al., 2018. Latent class analysis to evaluate performance of point-of-care CCA for low-intensity Schistosoma mansoni infections in Burundi. Parasit Vectors 11: 111.

23. Jordan P, 1985. Schistosomiasis: the St. Lucia Project. Cambridge, United Kingdom: Cambridge University Press.

24. Gaspard J et al., 2020. Survey of schistosomiasis in Saint Lucia: evidence for interruption of transmission. Amer J Trop Med Hyg. Available at: https://doi.org/10.4269/ajtmh.19-0904.

25. Maddison SE, Slemenda SB, Tsang VC, Pollard RA, 1985. Serodiagnosis of Schistosoma mansoni with microsomal adult worm antigen in an enzyme-linked immunosorbent assay using a standard curve developed with a reference serum pool. Am J Trop Med Hyg 34: 484-494.

26. Wilson S, Jones FM, van Dam GJ, Corstjens PL, Riveau G, Fitzsimmons CM, Sacko M, Vennervald BJ, Dunne DW, 2014. Human Schistosoma haematobium antifecundity immunity is dependent on transmission intensity and associated with immunoglobulin G1 to worm-derived antigens. J Infect Dis 210: 2009-2016.

27. Haggag AA, Rabiee A, Abd Elaziz KM, Gabrielli AF, Abdel Hay R, Ramzy RM, 2017. Mapping of Schistosoma mansoni in the Nile
Delta, Egypt: assessment of the prevalence by the circulating cathodic antigen urine assay. Acta Trop 167: 9-17.

28. Fenwick $A, 2017$. Schistosomiasis research and control since the retirement of Sir Patrick Manson in 1914. Trans R Soc Trop Med Hyg 111: 191-198.

29. Haggag AA, Rabiee A, Abd Elaziz KM, Campbell CH, Colley DG, Ramzy RMR, 2019. Thirty-day daily comparisons of Kato-Katz and CCA assays of 45 Egyptian children in areas with very low prevalence of Schistosoma mansoni. Am J Trop Med Hyg 100: 578-583.

30. Haggag AA, Casacuberta Partal M, Rabiee A, Abd Elaziz KM, Campbell CH, Colley DG, Ramzy RMR, 2019. Multiple praziquantel treatments of Schistosoma mansoni egg-negative, CCA-positive schoolchildren in a very low endemic setting in Egypt do not consistently alter CCA results. Am J Trop Med Hyg 100: $1507-1511$

31. Walters B, 2016. Migration, land use and forest change in St. Lucia, West Indies. Land Use Policy 51: 290-300.

32. Walters B, 2016. St. Lucia's tourism landscapes: economic development and environmental change in the West Indies. Caribbean Geogr 20: 3-21.

33. Ruberanziza EKM, Ortu G, Kanobana K, Mupfasoni D, Ruxin J, Fenwick A, Nyatanyi T, Karema C, Munyaneza T, Polman K, 2015. Nkombo island: the most important schistosomiasis mansoni focus in Rwanda. Am J Life Sci 3: 27-31.

34. Ruxin J, Negin J, 2012. Removing the neglect from neglected tropical diseases: the Rwandan experience 2008-2010. Glob Public Health 7: 812-822.

35. Krauth SJ, Coulibaly JT, Knopp S, Traore M, N'Goran EK, Utzinger $\mathrm{J}, 2012$. An in-depth analysis of a piece of shit: distribution of Schistosoma mansoni and hookworm eggs in human stool. PLoS Negl Trop Dis 6: e1969.

36. Cavalcanti MG, Cunha AFA, Peralta JM, 2019. The advances in molecular and new point-of-care (POC) diagnosis of schistosomiasis pre- and post-praziquantel use: in the pursuit of more reliable approaches for low endemic and non-endemic areas. Front Immunol 10: 858

37. Viana AG et al., 2019. Discrepancy between batches and impact on the sensitivity of point-of-care circulating cathodic antigen tests for Schistosoma mansoni infection. Acta Trop 197: 105049.

38. Ashton RA, Stewart BT, Petty N, Lado M, Finn T, Brooker S, Kolaczinski JH, 2011. Accuracy of circulating cathodic antigen tests for rapid mapping of Schistosoma mansoni and S. haematobium infections in southern Sudan. Trop Med Int Health 16: 1099-1103.

39. Rubaba O, Chimbari MJ, Soko W, Manyangadze T, Mukaratirwa $S, 2018$. Validation of a urine circulating cathodic antigen cassette test for detection of Schistosoma haematobium in Mkhanyakude district of South Africa. Acta Trop 182: 161-165.

40. Sanneh B et al., 2017. Field evaluation of a schistosome circulating cathodic antigen rapid test kit at point-of-care for mapping of schistosomiasis endemic districts in the Gambia. PLoS One 12: e0182003.

41. Stothard JR et al., 2009. An evaluation of urine-CCA strip test and fingerprick blood SEA-ELISA for detection of urinary schistosomiasis in schoolchildren in Zanzibar. Acta Trop 111: 64-70.

42. Savioli L, Hatz C, Dixon H, Kisumku UM, Mott KE, 1990. Control of morbidity due to Schistosoma haematobium on Pemba Island: egg excretion and hematuria as indicators of infection. Am J Trop Med Hyg 43: 289-295.

43. Siongok TKA, Ouma JH, Houser HB, Warren KS, 1978. Quantification of infection with Schistosoma haematobium in relation to epidemiology and selective population chemotherapy. II mass treatment with a single oral dose of metrifonate. J Infect Dis 138: 856-858.

44. Corstjens PLAM et al., 2020. Circulating Anodic Antigen (CAA): a highly sensitive diagnostic biomarker to detect active Schistosoma infections-improvement and use during SCORE. Am J Trop Med Hyg 103 (Suppl 1): 50-57.

45. Ochodo EA, Gopalakrishna G, Spek B, Reitsma JB, van Lieshout L, Polman K, Lamberton P, Bossuyt PM, Leeflang MM, 2015. Circulating antigen tests and urine reagent strips for diagnosis of active schistosomiasis in endemic areas. Cochrane Database Syst Rev 2015: CD009579. 